\title{
Probing the puzzle of persistent pests
}

Crop rotation is an ancient and ecologically benign agricultural technique that limits pest populations while replenishing depleted soil. It is still common today, and corn farms in the US and Europe often rotate between planting corn and soybeans to confound the western corn rootworm (WCR), Diabrotica virgifera virgifera, which devastates corn plants but generally cannot digest soy. Recently, however, some populations of WCR have shown resilience to crop rotation, feeding and ovipositing on soybeans as well as corn.

Scientists are still exploring the mechanisms behind this adaptation, and some research indicates that the gut microbiota facilitates soybean digestion in rotationresistant WCRs. Now, a team of researchers led by Manfredo Seufferheld (University of Illinois, Champaign) has turned to genetics to better understand this adaptation (Evol. Appl. doi:10.1111/eva.12278; published online 28 May 2015).

"The evolution of resistance in insects is complex," Seufferheld said in a press release; "human activity, the evolutionary history of the insects, the host and non-host plants, the microbial community and the genes all play a role."

Seufferheld's team collected WCRs from several populations with different phenotypes, including wild-type and rotationresistant, and fed different diets to WCRs of each phenotype. They provided some with a typical corn diet and others with soybean diets and then removed their guts for analysis.

The researchers found that wild-type and rotation-resistant WCRs expressed similar genes in their guts when both types were fed corn, but different genes when fed soybean diets. In total, they identified 3,973 genes that are differentially expressed in the guts of wild-type and rotation-resistant WCRs in at least one dietary condition.

In their analysis, they determined that many of these genes are associated with assorted physiological processes including growth and immune responses. "We also found differentially regulated genes involved in detoxification and genes involved in transport of metabolic products, lipids, sterols and drugs in and out of the cell," Seufferheld said.

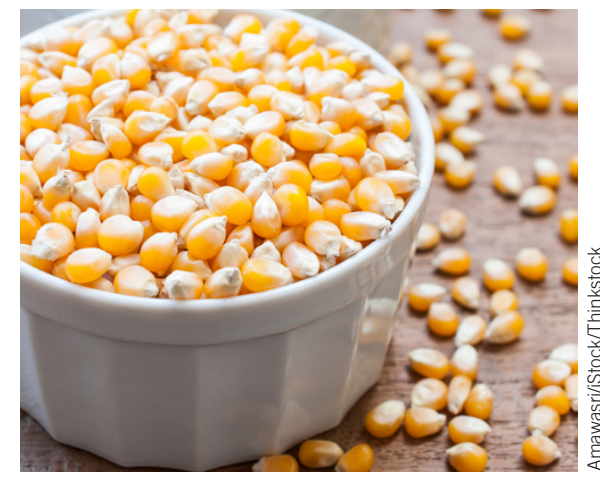

The emerging picture provides no immediate and simple explanation of rotation resistance in WCRs, but it illustrates the complex situational adaptations that can create a resistant population. Though WCRs are a particular bane of the agricultural industry, the evolution of resistance is a topic of concern of many fields. As conventional measures for biological control yield increasingly resilient pests in fields, barns, labs and hospitals, researchers must continue to investigate how resistance emerges in controlled populations.

Gregory D. Larsen

\section{HITTING A NERVE TO EASE ASTHMA}

Asthma is characterized by excessive sensitivity of the lungs to stimuli (including infections, allergens and irritants) leading to inflammation of the airway and difficulty breathing. Millions of people worldwide suffer from asthma, and its prevalence is growing, making asthma a global public health concern.

There is no cure for asthma, although pharmaceutical intervention can help to manage symptoms by suppressing inflammation or dilating the airway to ease breathing. "Current asthma treatments can help to control symptoms and dampen airway inflammation; however, therapies are not available to promote the resolution of asthma," said Bruce Levy, of Brigham and Women's Hospital (Boston, MA) in a press release. Now, results of a recent study led by Levy and Clifford Woolf, of Children's Hospital Boston and Harvard Medical School (Boston, MA), point toward a new approach to treating asthma. The results suggest that disabling a specific set of sensory neurons in the lungs, called nociceptors, can reduce airway inflammation and lung hypersensitivity (Neuron doi:10.1016/j.neuron.2015.06.007; published online 25 June 2015).

Nociceptors in the lungs trigger protective reflexes such as coughing in response to stimuli; nociceptors are also more abundant and more sensitive in people with asthma. Levy and Woolf's research team found that airway inflammation activates lung nociceptors and that this activation elicits a cascade of immune signals that intensifies inflammation and aggravates symptoms of asthma. The findings led to the hypothesis that interfering with nociceptor activation might have therapeutic benefits in asthma. The scientists tested this idea in mouse models of acute and chronic asthma by silencing nociceptors either genetically or pharmacologically. Silencing of nociceptors substantially reduced airway inflammation and lung hypersensitivity in the mice.

The pharmacological compound tested in the study is called QX-314. It is related to lidocaine, which is used as a local anesthetic because it blocks nerve signaling. Lidocaine has been tested as an asthma treatment, but it can have adverse outcomes because it affects all neurons. QX-314 is modified such that it specifically targets nociceptors that are activated by inflammation, including those in the lung, and does not readily enter the bloodstream. These properties reduce the compound's potential to cause side effects and extend its duration of action, improving its prospects for treating asthma.

"We are sufficiently encouraged by the strong relief of lung inflammation and airway constriction to actively embark on a major drug development program, with the aim of clinically testing this strategy [QX-314] for multiple allergic conditions," said Woolf in a press release. 